\title{
MicroRNA-1291 promotes endometrial fibrosis by regulating the ArhGAP29-RhoA/ROCK1 signaling pathway in a murine model
}

\author{
QIAN XU, HUA DUAN, LU GAN, XIN LIU, FANG CHEN, XUE SHEN, YI-QUN TANG and SHA WANG \\ Department of Gynecology Minimally Invasive Center, Beijing Obstetrics and Gynecology Hospital, \\ Capital Medical University, Dong Cheng, Beijing 100006, P.R. China
}

Received October 24, 2016; Accepted June 8, 2017

DOI: $10.3892 / \mathrm{mmr} .2017 .7210$

\begin{abstract}
Intrauterine adhesions (IUAs) are caused by endometrial damage and are associated with a poor pregnancy prognosis including infertility, oligomenorrhea and recurrent pregnancy loss. Understanding the pathogenesis of IUAs may help prevent and treat this condition more effectively. The aim of the current study was to investigate the function of microRNA-1291 (miR-1291) during the development of IUAs following endometrial damage and elucidate the potential molecular mechanisms involved. The expression of Rho GTPase activating protein 29 (ArhGAP29), a putative target mRNA of miR-1291, was determined by immunohistochemical staining of human endometrial tissue from patients with IUAs and compared with normal endometrial tissues. ArhGAP29 expression was significantly decreased in endometrial tissues with IUAs compared with normal endometrium. Additionally, a murine IUAs model was developed and reverse transcription-quantitative polymerase chain reaction (RT-qPCR) demonstrated that miR-1291 levels were significantly increased in the uterine tissue and plasma of the IUAs group compared with the normal mice. Furthermore, an miR-1291 antagomir was injected into the uterine cavity of experimental IUAs mice to block miR-1291. Hematoxylin and eosin and Masson's stain revealed that blocking miR-1291 significantly ameliorated endometrial fibrosis. Furthermore, levels of epithelial mesenchymal transition (EMT)-associated proteins, and ArhGAP29-RhoA/Rho-associated coiled coil containing protein kinase 1 (ROCK1) were measured in uterine tissue by western blot, RT-qPCR analysis and immunofluorescence staining. Levels of the mesenchymal marker proteins,
\end{abstract}

Correspondence to: Dr Hua Duan, Department of Gynecology Minimally Invasive Center, Beijing Obstetrics and Gynecology Hospital, Capital Medical University, 17 Qi Helou Street, Dong Cheng, Beijing 100006, P.R. China

E-mail: duanhuasci@163.com

Key words: intrauterine adhesions, fibrosis, epithelial mesenchymal transition, Rho GTPase activating protein 29, RhoA/Rho-associated coiled coil containing protein kinase 1, murine intrauterine adhesions model vimentin and $\mathrm{N}$-cadherin, were increased in the IUAs group mice, accompanied by a relative decrease in the epithelial marker proteins, cytokeratin and E-cadherin compared with normal murine endometrium. miR-1291 inhibition decreased RhoA/ROCK1 expression in the EMT pathway, but increased ArhGAP29 expression. Taken together, the findings indicate that miR-1291 acts upstream of ArhGAP29 to negatively regulate the RhoA/ROCK1 EMT pathway, ultimately leading to endometrial fibrosis. These studies may provide new potential therapeutic options and pave the way to use circulating miR-1291 as a clinical biomarker of endometrial fibrosis.

\section{Introduction}

Intrauterine adhesions (IUAs), also referred to as Asherman's syndrome, are characterized by the development of scar tissue inside the uterus or cervix leading to partial or complete adhesion of the endometrial surface. The most common cause of IUAs is uterine surgery (1) and the main clinical manifestations associated with this condition include hypomenorrhea, amenorrhea, periodic abdominal pain, abnormal pregnancy or secondary infertility (2-4). Once IUAs have developed, they are notoriously resistant to a variety of therapies, and prognosis is poor for many patients. Women of reproductive age who suffer from this disease may still undergo infertility or recurrent pregnancy loss, even after a long course of therapy. Although it is widely accepted that the disease is caused by damage incurred by the basal layer of the endometrium, there are many hypotheses regarding IUAs pathogenesis, including fibrocyte hyperplasia, which leads to the over-deposition of extracellular matrix and proliferation of fibrous connective tissue (5). However, the precise pathological mechanisms involved remain unclear.

MicroRNAs (miRNAs) are non-coding, single-stranded, RNA molecules that are 20-22 nucleotides in length. It has been previously demonstrated that miRNAs are able to inhibit gene expression at the post-transcriptional level by nucleotide base pairings between complementary sequences of miRNAs and the 3'-untranslated region (3'-UTR) (6). A number of miRNAs have been identified to have an important role in fibrosis in a variety of cell types, including miRNAs in hepatic, renal, cardiac and oral sub-mucous tissues (7-10). Recent studies have also highlighted the regulatory function of miR-29b in endometrial fibrosis via the tumor growth factor (TGF)- $\beta 1 / \mathrm{Smad}$ 
epithelial mesenchymal transition (EMT) pathway; however, the precise association between the EMT pathway and the development of endometrial fibrosis remains unclear (11). Our previous study used gene-chips to identify the differential expression of miRNAs in the normal and IUAs human endometrium samples (12); it was demonstrated that the miRNA (miR)-1291 level was significantly increased in endometrium affected by IUAs. miR-1291 has previously been demonstrated to perform a variety of functions, including the modulation of cellular drug disposition and chemosensitivity, and regulation of cell proliferation and metabolism by targeting specific sets of genes (13-15). However, the molecular mechanisms underlying the important functions have yet to be elucidated.

A range of analyses and predictions, using gene ontology (GO) consortium and MiRDB databases, along with pathway prediction and Targetscan software, recently identified Rho GTPase activating protein 29 (ArhGAP29) as a target gene for miR1291 (12). ArhGAP29 is a specific type of RhoGTPase activation protein (GAP) known to inhibit the Rho signaling pathway (16-19). A variety of studies have investigated the association between ArhGAP29 and Rho protein. Notably, depletion of ArhGAP29 can affect functional activity of the endothelial barrier leading to increased endothelial permeability, while depletion of Rho proteins can have the opposite effect (17). However, the precise molecular mechanisms underlying the role of ArhGAP29 in IUAs have yet to be explained.

Due to the difficulty in obtaining appropriate tissue from the human endometrium, a murine IUAs model was established for the experiments in the current study. The study had three objectives: i) To explore differences in miR-1291 levels and EMT-associated proteins in uterine tissue from normal mice and those with IUAs; ii) to determine whether miR-1291 inhibition can ameliorate endometrial fibrosis; and iii) to elucidate whether miR-1291 affects the fibrotic RhoA/Rho-associated coiled-coil containing protein kinase 1 (ROCK1) EMT pathway via regulation of ArhGAP29.

\section{Materials and methods}

Patients samples and immunohistochemistry. The subjects in the current study included 39 patients with IUAs diagnosed by hysteroscope and 28 normal control cases recruited between July 2014 and June 2015 from the Beijing Obstetrics and Gynecology Hospital. Cases selected were all women of reproductive age. The age of the patients with IUAs ranged from 23-40 years old, while ages in normal control group ranged from 27-40. Normal control endometrial tissues were collected from patients who underwent hysteroscopies due to infertility or other factors during the same period. Formalin-fixed, paraffin-embedded endometrial tissues were obtained from the Department of Pathology in Beijing Obstetrics and Gynecology Hospital (Beijing, China) and were originally obtained as pre-therapeutic biopsies. The study was approved by the Capital Medical University Research Ethics Committee (Beijing, China) following the principles of the Helsinki Declaration, and all participants provided informed consent. Immunohistochemistry, using a commercial primary anti-ArhGAP29 antibody (NBP1-05989; Novus Biologicals Canada ULC, Oakville, ON, Canada), RhoA (sc179; 1:50; Santa Cruz Biotechnology, Inc., Dallas, TX, USA) and
ROCK1 (ab45171; 1:200; Abcam, Cambridge, MA, USA) were used to investigate the differential expression of ArhGAP29 and RhoA/ROCK1 in severe IUAs compared with normal endometrial tissue.

Animals. A total of 12 mice (female C57BL/6 strain) were provided by the Experimental Animal Center of Beijing Chaoyang Hospital, Capital Medical University (Beijing, China). All experimental mice were 8 weeks of age, weighing $19.4 \pm 0.45 \mathrm{~g}$ and were housed in a temperature-controlled room $\left(22.2^{\circ} \mathrm{C}\right)$ with a constant 12 -h light/dark cycle. Each cage contained 4 mice with ad libitum access to food. The experiments were approved by the Animal Experiment Ethics Committee of Capital Medical University, and all animals were treated using a protocol approved by the Capital Medical University Institute of Animal Care. All tissues were examined in a blinded manner in order to remove the risk of potential bias.

Establishing an IUAs murine model and application of miR-1291 antagomir. All experimental mice were anesthetized with $5 \%$ pentobarbital sodium $(1 \mathrm{ml} / \mathrm{kg})$ via intraperitoneal injection. A vertical incision was then made in the abdominal wall in order to expose the uterus. A small incision was then made in each uterine horn at the uterotubal junction and the horns were traumatized using standardized methodology via a 27 Gauge needle inserted two-thirds of the way through the lumen, rotated and withdrawn four times to establish a IUAs murine model (20). A mouse miR-1291 antagomir (5'-AAC UGCUAGUCUUCAGUAAGAGCCAU-3'), and an appropriate negative control (NC; 5'-CAGUACUUUUGUGUA GUACAAA-3') were chemically synthesized by Guangzhou RiboBio Co., Ltd. (Guangzhou, China). Mice were divided into three groups: IUAs $(n=3)$, miR-1291 antagomir $(n=3)$ and $\mathrm{NC}(\mathrm{n}=3)$. Following surgery, $5 \mathrm{nmol} \mathrm{miR}-1291$ antagomir or $\mathrm{NC}$ was injected into the musculature of each side of the damaged murine uterine horns to establish the miR-1291 antagomir group or $\mathrm{NC}$ group. After 7 days, the uterine horns were collected for tissue staining, gene expression studies and analysis of protein levels.

Histological staining of murine tissue. Murine endometrial tissue from normal controls and from animals with IUAs were formalin-fixed at room temperature for $48 \mathrm{~h}$ and then paraffin-embedded. Thin sections $(5 \mu \mathrm{m})$ were then cut and stained by hematoxylin and eosin (H\&E) and Masson's trichrome. H\&E staining: Sections were stained in hematoxylin (1:200 dilution) for $45 \mathrm{sec}$, and stained in eosin (1:400 dilution) at room temperature for $60 \mathrm{sec}$. Masson staining: Sections were stained in hematoxylin at $60^{\circ} \mathrm{C}$ in a hot water bath for 5 min, Ponceau Acid Fuchsin for 5-10 min and aniline blue solution for $5 \mathrm{~min}$ at room temperature (Beijing Zhongshan Golden Bridge Biotechnology, Co., Ltd., Beijing, China). H\&E was used to investigate changes in the endometrium structure or number of endometrial glands, Masson's trichrome was used to determine the thickness and degree of endometrial collagen deposition. The extent of fibrosis was also calculated in order to compare the degree of endometrial fibrosis between experimental groups. Four random fields of each Masson's trichrome slide were chosen to calculate the ratio of endometrial fibrotic 
area/total endometrial areas (the uterine cavity was excluded). All images were captured using an Olympus microscope (SP 500; Olympus Corporation, Tokyo, Japan) and analyzed using ImageJ analysis software version 1.50 (National Institutes of Health, Bethesda, MD, USA; https://imagej.nih.gov/ij/).

Immunofluorescence staining. Uterine horns collected from either miR-1291 antagomir or NC treated mice were frozen in Tissue-Tek ${ }^{\circledR}$ O.C.T. Compound and sectioned into $9 \mu \mathrm{m}$ pieces for immunofluorescence staining. Non-specific antigens in the sections were blocked using $10 \%$ goat serum (Beijing Zhongshan Golden Bridge Biotechnology Co. Ltd.) for $1 \mathrm{~h}$ following antigen retrieval. Antigen retrieval was performed in a pressure cooker using EDTA (1:50 dilution, $\mathrm{pH} 9.0$ ) for $20 \mathrm{~min}$ in boiling water. Sections were then incubated overnight at $4^{\circ} \mathrm{C}$ with primary antibodies raised against ArhGAP29 (NBP1-05989; 1:50; Novus Biologicals Canada ULC), RhoA (sc179; 1:100; Santa Cruz Biotechnology, Inc.) and ROCK1 (ab45171; 1:100; Abcam). Subsequently, Alexa Fluor 488 goat anti-rabbit IgG antibody (A11008; 1:200; Thermo Fisher Scientific, Inc.) was used as a secondary antibody and incubated with the sections for $1 \mathrm{~h}$ at room temperature. Nuclei were stained with 4,6-diamidino-2-phenylindole (DAPI, $200 \mathrm{ng} / \mathrm{ml}$ ) for $10 \mathrm{~min}$ at room temperature. Images were captured using an Olympus microscope (IX51; Olympus Corporation, Tokyo, Japan).

Reverse transcription-quantitative polymerase chain reaction (RT-qPCR). For each experimental animal, endometrial tissue was macerated in a porcelain mortar and blood plasma was prepared from $6 \mathrm{ml}$ of EDTA-treated blood. Total RNA was extracted from tissues and plasma using RNAiso Plus (AKA1304; Takara Bio, Inc., Otsu, Japan) separately according to the manufacturer's protocols. RNA quality was confirmed using a NanoDrop 2000 spectrophotometer (Thermo Fisher Scientific, Inc.). RT was then performed using the FastQuant RT cDNA synthesis kit (Tiangen Biotech Co., Ltd., Beijing, China). A cDNA library of miRNAs expressed by the endometrial tissues was also constructed using a miRcute miRNA cDNA synthesis kit (Tiangen Biotech Co., Ltd.) and a miRNA qPCR Starter kit (Guangzhou RiboBio Co., Ltd.) according to the manufacturer's protocols. The mRNAs and miRNAs were then amplified via a SYBR-Green reaction using a SuperReal PreMix Plus and miRcute miRNA qPCR Detection kit (Tiangen Biotech Co., Ltd.) and an ABI 7500 RT-qPCR system (Applied Biosystems; Thermo Fisher Scientific, Inc.) according to the manufacturer's protocols. The mRNAs cycling parameters for PCR were as follows: an initial denaturation at $95^{\circ} \mathrm{C}$ for $15 \mathrm{~min}$, followed by 40 cycles of denaturation at $95^{\circ} \mathrm{C}$ for $10 \mathrm{sec}$, annealing at $55^{\circ} \mathrm{C}$ for $20 \mathrm{sec}$ and extension at $72^{\circ} \mathrm{C}$ for $32 \mathrm{sec}$. And the miRNAs cycling parameters for PCR were as follows: denaturation at $94^{\circ} \mathrm{C}$ for $2 \mathrm{~min}$, followed by 40 cycles of annealing at $94^{\circ} \mathrm{C}$ for $20 \mathrm{sec}$ and extension at $60^{\circ} \mathrm{C}$ for $34 \mathrm{sec}$. Relative gene expression was examined using the comparative $\mathrm{Cq}$ method with GAPDH as an endogenous control to normalize data (21). U6 small nuclear RNA was used as an endogenous control to normalize the relative expression levels of miR-1291. To ensure technical reliability, experiments were run in triplicate for each case. Primers for RT-qPCR are listed in Table I.
Table I. Primer list for polymerase chain reaction.

Gene $\quad$ Sequence $\left(5^{\prime}-3^{\prime}\right)$

$\begin{array}{ll}\text { Cytokeratin } & \\ \text { Forward } & \text { TGGTGGTTTTGGTAGTGGA } \\ \text { Reverse } & \text { AGGCAAACTTGTCATTGAGTGAC }\end{array}$

E-cadherin

Forward

GACCGAGAGAGTTACCCT

Reverse

CTTGACCCTGATACGTGC

N-cadherin

Forward

Reverse

GCAGTCTTACCGAAGGATGTG

GAAATCTGCTGGCTCGCT

Vimentin

Forward

Reverse

TCTACCAGGTCTGTGTCC

Collagen,

type I, $\alpha 1$

Forward

Reverse

GGGGGATGAGGAATAGAG

$\alpha$-Smooth

muscle actin

Forward

Reverse

TGACAGAGGCACCACTGAAC

Fibroblast

growth factor 2

Forward

Reverse

CCCTGAAGTCAGCTGCATA
GGCAGAAAGCACAGCACTC

Platelet derived

growth factor BB

Forward

Reverse

ATGAAATGCTGAGCGACC

ATTACAGCAGGCTCTGCT

Rho GTPase

activating protein 29

Forward

CATCCCTTGCCAACAGTTTACAGT

Reverse

TCAGCAGGTTCATAGCCAGATG

RhoA

Forward

CTGCCATCAGGAAGAAAC

Reverse

ATCCACCTCGATATCCGC

Rho-associated

coiled-coil

containing

protein kinase 1

Forward

TGAGGCATAAATCCACTAG

Reverse

AAGGACTATTGGCAAACG

GAPDH

Forward

Reverse

GTCCCAGCTTAGGTTCAT ATCTCCACTTTGCCACTG

Western blot analysis. Endometrial tissues were prepared for protein detection by maceration with radioimmunoprecipitation lysis buffer (Beijing GENIA Biotechnology Co., Ltd., Beijing, China) in a porcelain mortar. Protein concentration was then determined using the bicinchoninic acid protein assay 
Table II. Primary antibodies used in western blot analysis.

\begin{tabular}{|c|c|c|c|c|c|}
\hline Antibody & Source & Type & Dilution & Cat. no. & Company \\
\hline E-cadherin & Rabbit & Polyclonal & $1: 800$ & sc7870 & $\begin{array}{l}\text { Santa Cruz Biotechnology, Inc. } \\
\text { (Dallas, TX, USA) }\end{array}$ \\
\hline Cytokeratin & Rabbit & Polyclonal & $1: 500$ & sc15367 & Santa Cruz Biotechnology, Inc. \\
\hline N-cadherin & Rabbit & Monoclonal & $1: 4,000$ & $\mathrm{ab} 76011$ & Abcam (Cambridge, MA, USA) \\
\hline Vimentin & Mouse & Monoclonal & $1: 500$ & sc373717 & Santa Cruz Biotechnology, Inc. \\
\hline$\alpha$-Smooth muscle actin & Rabbit & Monoclonal & $1: 1,000$ & ab32575 & Abcam \\
\hline Collagen, type I, $\alpha 1$ & Rabbit & Polyclonal & $1: 1,000$ & ab34710 & Abcam \\
\hline Fibroblast growth factor 2 & Rabbit & Polyclonal & $1: 500$ & ab16828 & Abcam \\
\hline Platelet-derived growth factor BB & Rabbit & Polyclonal & $1: 500$ & ab23914 & Abcam \\
\hline Rho GTPase activating protein 29 & Rabbit & Polyclonal & $1: 2,000$ & NBP1-05989 & $\begin{array}{l}\text { Novus Biologicals Canada ULC } \\
\text { (Oakville, ON, Canada) }\end{array}$ \\
\hline RhoA & Rabbit & Polyclonal & $1: 400$ & sc179 & Santa Cruz Biotechnology, Inc. \\
\hline $\begin{array}{l}\text { Rho-associated coiled-coil } \\
\text { containing protein kinase } 1\end{array}$ & Rabbit & Monoclonal & $1: 500$ & ab45171 & Abcam \\
\hline GAPDH & Rabbit & Monoclonal & $1: 2,000$ & ab181602 & Abcam \\
\hline
\end{tabular}

kit (Kangwei Century Biotechnology Co., Ltd., Beijing, China) and an equal amount of protein (30 $\mu \mathrm{g}$ in each lane) was loaded and separated on $10 \%$ SDS-PAGE gels under denaturing conditions. Proteins were then transferred to polyvinylidene fluoride membranes (EMD Millipore, Billerica, MA, USA), blocked in 5\% non-fat milk at room temperature for $30 \mathrm{~min}$ and incubated with the specific primary antibodies at $4{ }^{\circ} \mathrm{C}$ overnight (Table II). Equivalent protein loading and transfer efficiency were verified by GAPDH staining. Following incubation at room temperature for $20 \mathrm{~min}$ with appropriate horseradish peroxidase-conjugated secondary $\mathrm{IgG}$ antibodies (AS003/AS014; ABclonal Biotech Co., Ltd., Wuhan, China), antigen detection was performed using an enhanced chemiluminescence detection system (WBKLS100; EMD Millipore). Images were acquired using a ChemiDoc XRS+ system (Bio-Rad Laboratories, Inc., Hercules, CA, USA) and subsequently analyzed with ImageJ software.

Statistical analysis. All data are presented as the mean \pm standard deviation of at least three replicates. SPSS software version 19.0 (IBM Corp., Armonk, NY, USA) was used for data analysis. Differences between two groups were analyzed using the Student's t test. One-way analysis of variance was used when comparisons included three or more groups. Multiple comparison between the groups was performed using LSD method. $\mathrm{P}<0.05$ was considered to indicate a statistically significant difference.

\section{Results}

Immunohistochemical staining of ArhGAP29-RhoA/ROCK1 in the human endometrium. Immunohistochemical staining was used to evaluate the expression of ArhGAP29-RhoA/ROCK1 in normal and severe IUAs human endometrium samples. ArhGAP29 staining was more obvious in normal endometrium compared with endometrial tissue with severe IUAs, while immunohistochemical staining of RhoA/ROCK1 in human endometrium with severe IUAs was more obvious than in normal endometrial tissue (Fig. 1). As ArhGAP29 is a putative target mRNA of miR-1291, this finding is in accordance with the increased levels of miR-1291 in tissues with severe IUAs (12).

Establishment and evaluation of a murine IUAs model. In order to determine the relative degree of uterine damage, 3 mice from each group were sacrificed 7 days after surgery and endometrial tissues were analyzed by H\&E and Masson's trichrome stain. Uterine endometrial structure was disordered, the number of glands was significantly reduced, and fibers were bulkier and more diffuse in the IUAs group compared with the normal control group (Fig. 2A and B). Masson's trichrome staining confirmed the presence of fibrosis in the uterine endometrium. Collagen fibers were stained blue, while muscle, vessels and mucosa were stained dark red. Masson's trichrome staining confirmed that the extent of fibrosis differed markedly between the two groups; the fibrotic area varied from 12 to $90 \%$ (Fig. 2C and D). The extent of fibrosis was significantly higher in the IUAs group compared with the normal control group $(\mathrm{P}<0.001$; Fig. $2 \mathrm{E})$, indicating that a murine IUAs model was successfully established.

Changes in miR-1291 and EMT-associated proteins during the IUAs development. RT-qPCR was used to evaluate differential expression of miR-1291 between normal controls and the IUAs group murine tissues. Data indicated that miR-1291 expression was significantly increased in both the plasma and uterine tissues from mice with IUAs. Indeed, miR-1291expression in endometrial tissue from the IUAs group was 7.9 fold higher than that of the normal control group $(\mathrm{P}<0.001$; Fig. $2 \mathrm{~F})$, and 4.2 fold higher in the plasma $(\mathrm{P}<0.01$; Fig. $2 \mathrm{~F})$. Relative changes in EMT-associated marker proteins in the IUAs group and normal controls were also assessed. RT-qPCR and western blot analysis revealed a reduction in the epithelial marker proteins cytokeratin and E-cadherin, accompanied 


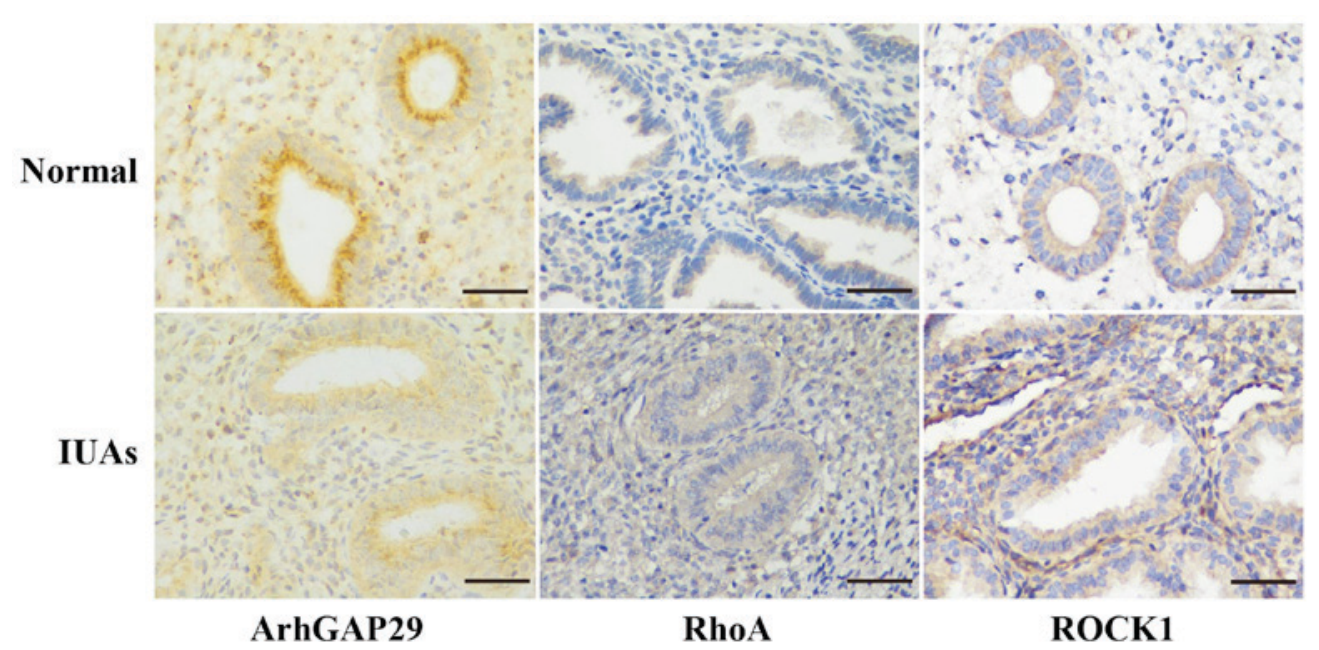

Figure 1. Immunohistochemical staining of ArhGAP29-RhoA/ROCK1 in human endometrium. ArhGAP29 staining in normal human endometrium was more obvious compared with endometrial tissue with severe IUAs; whereas, immunohistochemical staining of RhoA/ROCK1 in human endometrium with severe IUAs was more obvious compared with normal endometrial tissue. Scale bar, $50 \mu \mathrm{m}$. IUAs, intrauterine adhesions; ArhGAP29, Rho GTPase activating protein 29; ROCK1, Rho-associated coiled-coil containing protein kinase 1.
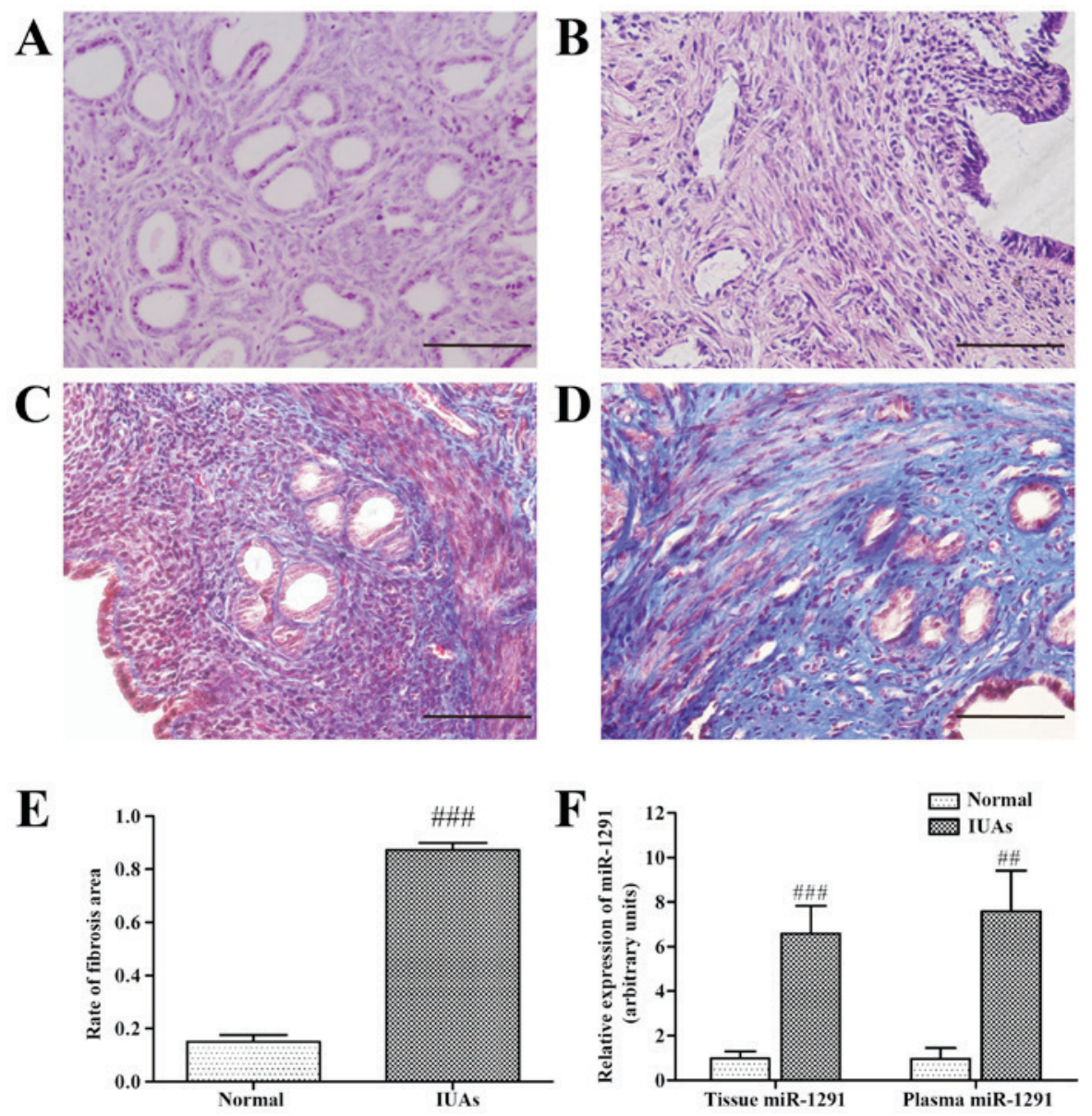

Figure 2. Establishment of a murine IUAs model and changes in miR-1291. Hematoxylin and eosin staining staining of (A) normal tissue and (B) IUAs uterine tissue. Masson's trichrome staining of uterine tissues in the (C) normal control group and (D) from the IUAs group. (E) The extent of fibrosis. (F) Expression of miR-1291 as measured by reverse transcription-quantitative polymerase chain reaction. ${ }^{\# \#} \mathrm{P}<0.01,{ }^{\# \# \#} \mathrm{P}<0.001$ vs. normal group. Scale bar, $50 \mu \mathrm{m}$. IUAs, intrauterine adhesions; miR, microRNA.

by an increase in the mesenchymal marker proteins vimentin and $\mathrm{N}$-cadherin in the IUAs group compared with the normal group (Fig. 3). Collectively, these results indicate that the EMT pathway may have a vital role in endometrial fibrosis.
Amelioration of endometrial fibrosis by miR-1291 antagomir. In order to block the function of miR-1291 during IUAs development, miR-1291 antagomir was injected into the murine uterus immediately following establishment of the murine IUAs 


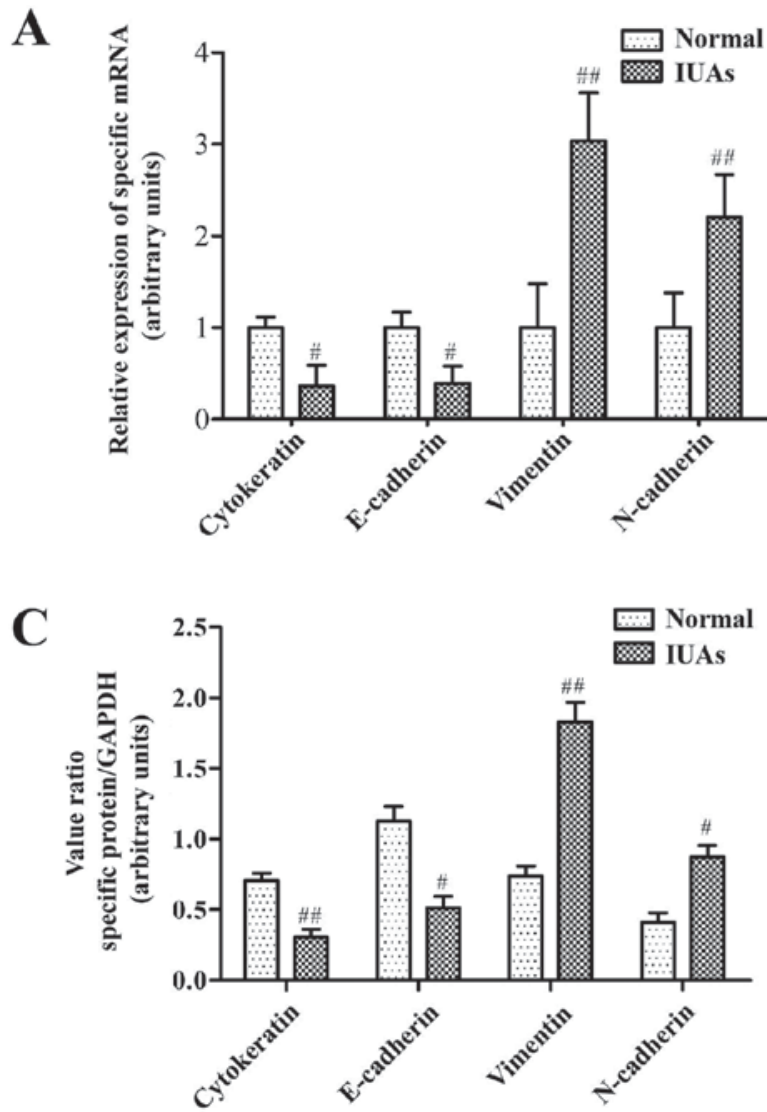

B

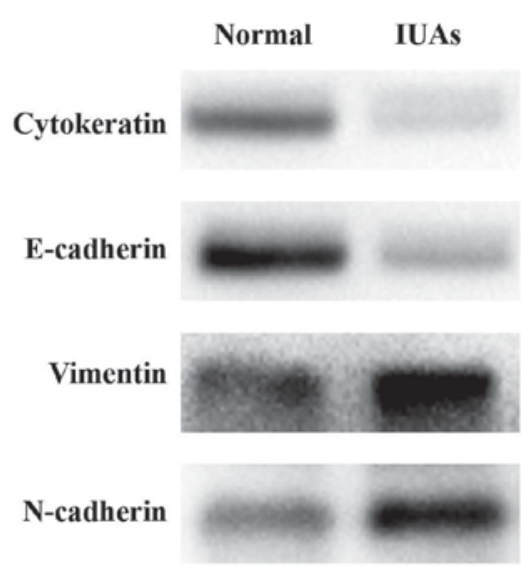

GAPDH

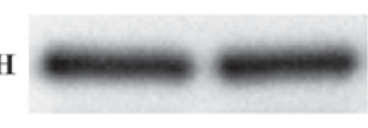

Figure 3. Changes of epithelial mesenchymal transition-associated proteins in a murine IUAs model. (A) Epithelial marker proteins, cytokeratin and E-cadherin and the mesenchymal marker proteins, vimentin and $\mathrm{N}$-cadherin, were measured by reverse transcription-quantitative polymerase chain reaction. (B) Representative images of western blot experiments. (C) Densitometry quantification of cytokeratin, E-cadherin, vimentin and N-cadherin by western blot analysis. ${ }^{\#} \mathrm{P}<0.05,{ }^{\# /} \mathrm{P}<0.01$ vs. normal group. IUAs, intrauterine adhesions.

model. For comparison, miR-1291 NC was injected into a second set of animals to determine whether miR-1291 affected endometrial fibrosis. At 7 days after surgery, animals were culled and uterine horns were collected for fibrosis evaluation. Masson's trichrome staining was used to investigate the extent of fibrosis amongst the IUAs group, the miR-1291 antagomir group and the NC group. The extent of fibrosis was clearly reduced in the miR-1291 antagomir group compared with the IUAs group and the NC group (Fig. 4). Furthermore, the relative expression of adhesion-associated proteins, including $\alpha$-smooth muscle actin $(\alpha$-SMA), collagen 1a, platelet derived growth factor (PDGF)-BB and fibroblast growth factor 2 (FGF2) was measured by RT-qPCR and western blot analysis in order to investigate their relative association with fibrosis alleviation. The data demonstrated that the expression of adhesion-associated proteins in the miR-1291 antagomir group was significantly lower than the IUAs group and the NC group (Fig. 5). Thus, the data suggest that miR-1291 may promote the IUAs development by aggravating endometrial fibrosis and that miR-1291 antagomir can ameliorate endometrial fibrosis.

miR-1291 antagomir inhibits the miR-1291/ArhGAP29RhoA/ROCK1 pathway. Previous studies have demonstrated that ArhGAP29 has a role in cell adhesion, cell to extracellular matrix adhesion, actin rearrangement and influences the progression of EMT-associated diseases $(18,22-24)$. In addition, ArhGAP29 is a key regulator of RhoA activity (19).
Therefore, it was hypothesized that miR-1291 influences the development of IUAs by regulating the RhoA/ROCK1 EMT pathway via the suppression of ArhGAP29.

Initially, the protein levels in endometrial tissues were investigated by immunofluorescence staining. ArhGAP29, RhoA and ROCK1 expression in the IUAs group, miR-1291 antagomir group and NC group were evaluated and compared. ArhGAP29 staining was stronger in the miR-1291 antagomir group compared with the IUAs group, while ArhGAP29 expression in the NC group demonstrated no marked difference compared to the IUAs group (Fig. 6). RhoA levels in the miR-1291 antagomir group were weaker than the IUAs group and the NC group (Fig. 6). There was no marked difference between the IUAs group and the NC group in terms of RhoA expression. The relative levels of ROCK1 followed the same pattern among groups as observed with RhoA expression (Fig. 6). Western blot and RT-qPCR analysis also indicated that the expression of ArhGAP29 was higher in the miR-1291 antagomir group compared with the other two groups, along with lower RhoA and ROCK1 expression (Fig. 7).

\section{Discussion}

The present study demonstrated that miR-1291 levels were significantly increased in the uterus and plasma in a murine IUAs model. Additionally, EMT occurred in the development 

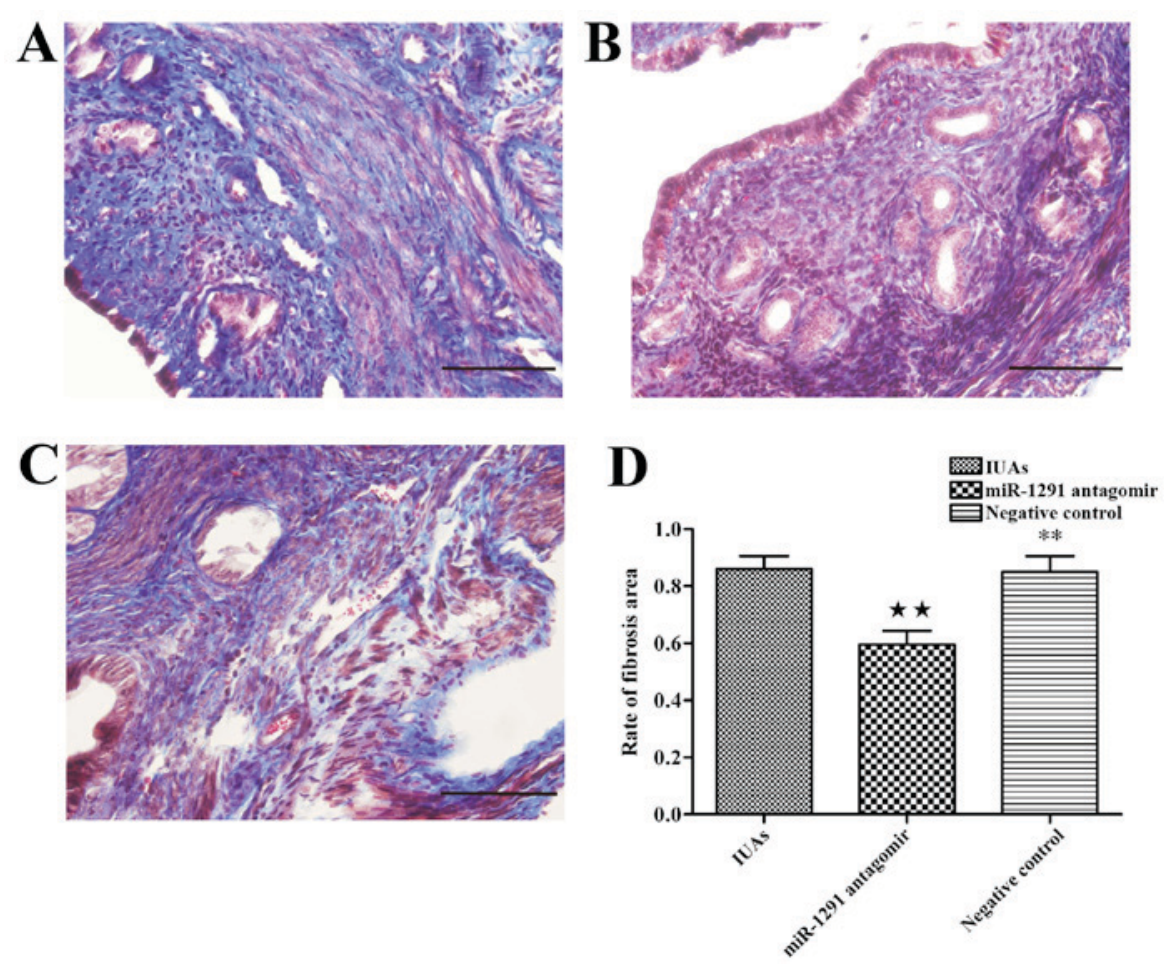

Figure 4. miR-1291 antagomir ameliorates endometrial fibrosis. Masson's trichrome staining in uterine tissues from the (A) IUAs group, (B) miR-1291 antagomir group and (C) the negative control group. (D) The extent of fibrosis in the IUAs group, miR-1291 antagomir group and the negative control group. ${ }^{\star \star} \mathrm{P}<0.01$ vs. IUAs group; ${ }^{* *} \mathrm{P}<0.01$ vs. miR-1291 antagomir group. IUAs, intrauterine adhesions; miR, microRNA.

A

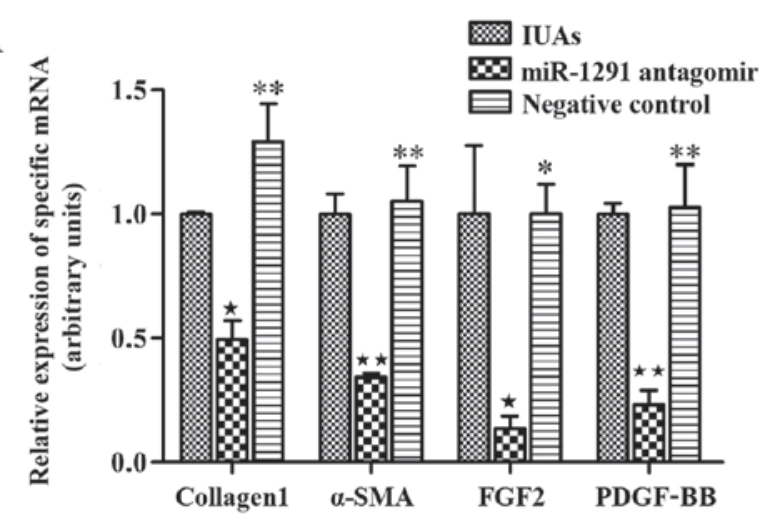

C

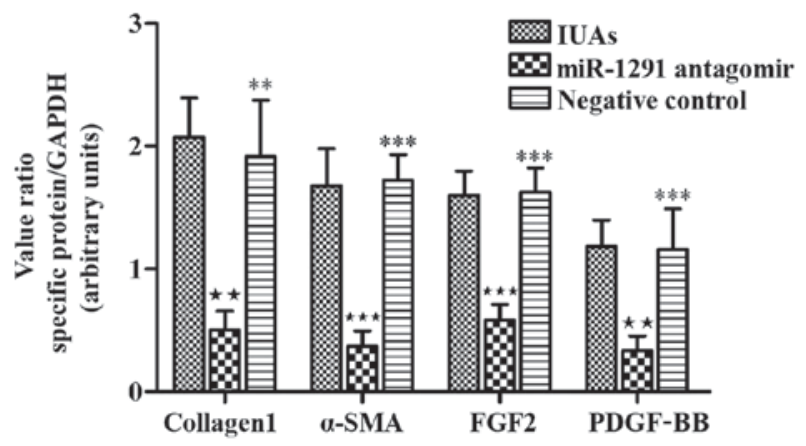

B

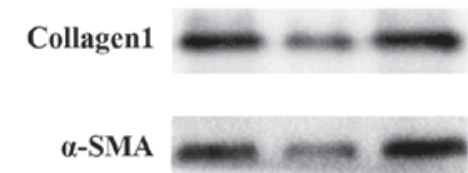

$\alpha$-SMA

FGF2

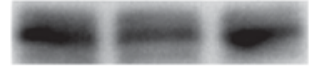

PDGF-BB

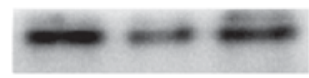

GAPDH

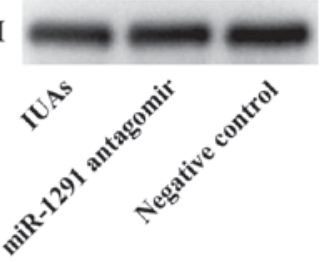

Figure 5. Decrease of adhesion-associated proteins by miR-1291 antagomir. (A) Expression levels of various adhesion-related proteins including $\alpha$-SMA, collagen 1, PDGF-BB and FGF2 were tested by RT-qPCR. (B) Representative western blotting images. (C) Densitometry analysis of the expression of $\alpha$-SMA, collagen 1, PDGF-BB and FGF2 by western blot analysis. ${ }^{\star} \mathrm{P}<0.05,{ }^{\star \star} \mathrm{P}<0.01,{ }^{\star \star \star} \mathrm{P}<0.001$ vs. IUAs group; ${ }^{*} \mathrm{P}<0.05,{ }^{* *} \mathrm{P}<0.01,{ }^{* * *} \mathrm{P}<0.001$ vs. miR-1291 antagomir group. IUAs, intrauterine adhesions; miR, microRNA; $\alpha$-SMA, $\alpha$-smooth muscle actin; FGF2, fibroblast growth factor 2; PDGF-BB, platelet derived growth factor-BB.

of endometrial fibrosis in the murine IUAs model. miR-1291 inhibition reduced expression of the small GTPase RhoA and its downstream partner, ROCK1 (25-27), along with the downregulated expression of ArhGAP29, a RhoA 


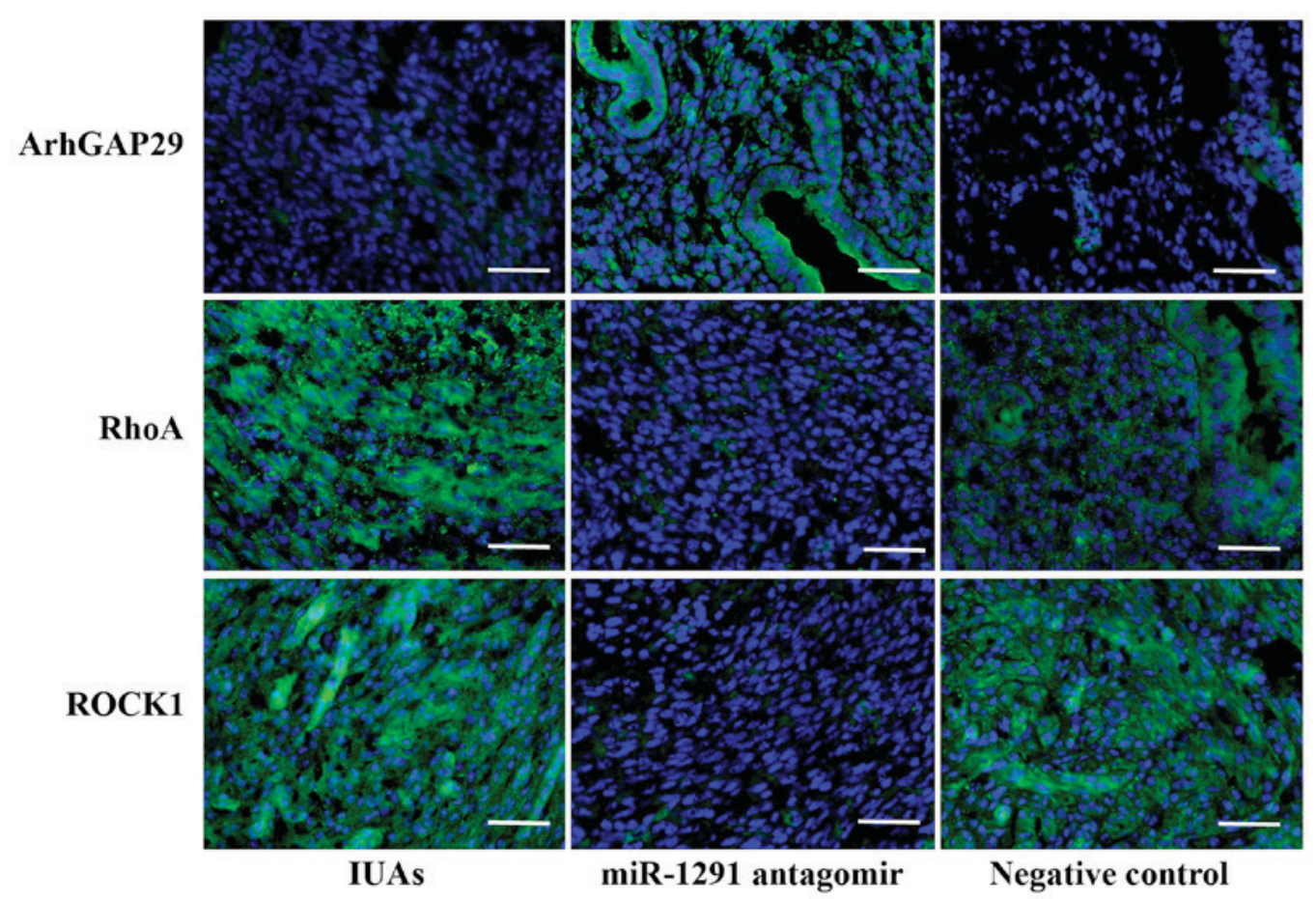

Figure 6. Effect of miR-1291 antagomir on ArhGAP29-RhoA/ROCK1 pathway in murine uterine tissue. Immunofluorescence staining for ArhGAP29, RhoA and ROCK1 (green) and nuclear DNA (DAPI, blue) in murine uterine tissue from the IUAs group, miR-1291 antagomir group and the negative control group. Scale bar, $50 \mu \mathrm{m}$. ArhGAP29, Rho GTPase activating protein 29; ROCK1, Rho-associated coiled-coil containing protein kinase 1; IUAs, intrauterine adhesions; miR, microRNA.

A

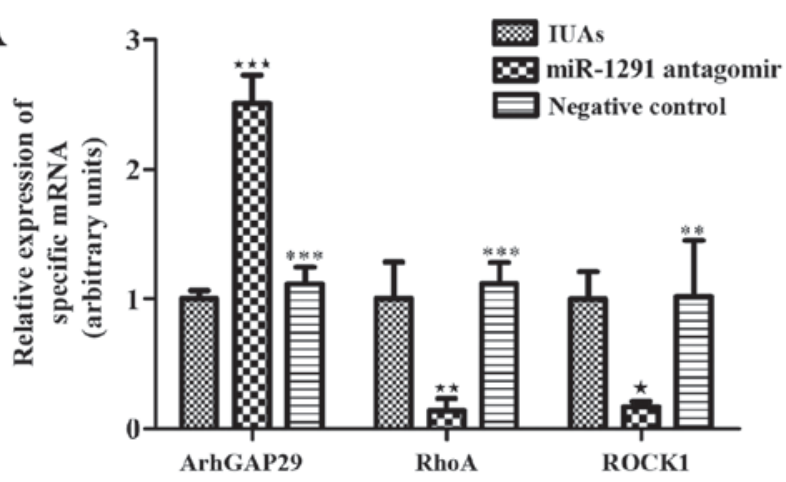

C

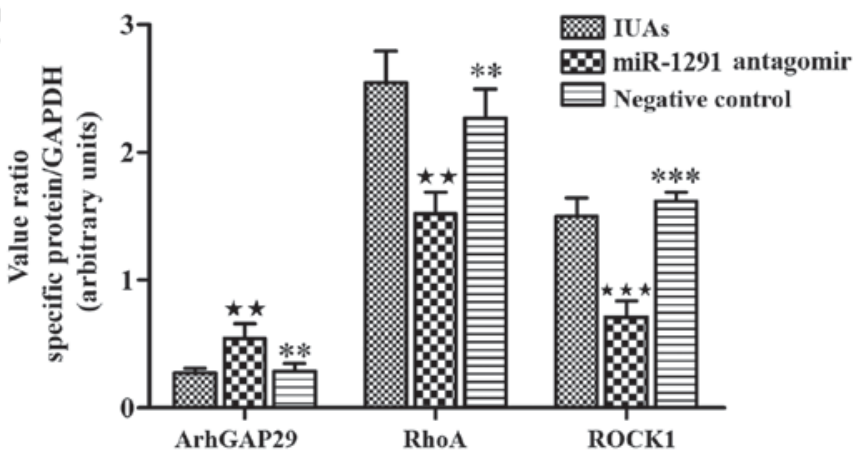

B

ArhGAP29

RhoA

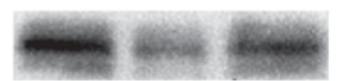

ROCK1

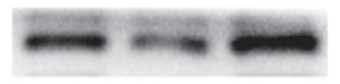

GAPDH

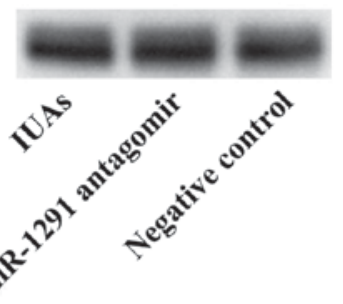

Figure 7. Effect of miR-1291 antagomir on ArhGAP29-RhoA/ROCK1 expression. (A) The expression levels of ArhGAP29, RhoA and ROCK1 were examined by reverse transcription-quantitative polymerase chain reaction. (B) Representative western-blot images and (C) densitometry of expression of ArhGAP29, RhoA and ROCK1 following western blotting analysis. ${ }^{\star} \mathrm{P}<0.05,{ }^{\star \star} \mathrm{P}<0.01$ vs. the IUAs group; ${ }^{* *} \mathrm{P}<0.01,{ }^{* * *} \mathrm{P}<0.001$ vs. miR-1291 antagomir group. ArhGAP29, Rho GTPase activating protein 29; ROCK1, Rho-associated coiled-coil containing protein kinase 1; IUAs, intrauterine adhesions; miR, microRNA.

inactivator (19). These molecular changes were accompanied by the reduced formation of endometrial fibrosis in the mouse model following administration of an miR-1291 inhibitor.
These observations suggest that the advent of IUAs is partially associated with an EMT-associated disease driven by the ArhGAP29-RhoA/ROCK1 pathway regulated by miR-1291. 
Our previous study of miRNA expression signatures in IUAs and normal endometrium demonstrated that miR-1291 was significantly upregulated in endometrial tissue with IUAs and that ArhGAP29 was the putative target gene. This suggested that miR-1291 has a role in endometrial fibrosis. As the miR-1291 level in the plasma was increased, the same trend as with tissue miR-1291 expression in the murine IUAs model, it follows that miR-1291 may represent a non-traumatic biomarker for the clinical diagnosis of endometrial fibrosis. However, while miR-1291 can modulate cellular reaction to drugs and regulate cell proliferation and migration (13-15), there has not yet been a specific investigation into the potential molecular mechanisms underlying the action of miR-1291 in IUAs development.

ArhGAP29 is a type of RhoGTPase activation protein (RhoGAP) and has a functional role in a range of cellular pathways by changing RhoGTPase activity. By regulating cell-cell adhesion and various components of the actin cytoskeleton, RhoGAP can change the stability of epithelial cell polarity, thus influencing the development and outcome of EMT-associated diseases (22-24). Studies have suggested that ArhGAP29 plays a vital role in modulating the cytoskeleton and actin rearrangement (18), however until now, its function in IUAs development had not been investigated.

Our previous study demonstrated that IUAs development is likely associated with EMT. In addition to miR-1291, other miRNAs that may be associated with the EMT pathway were also identified, including miR-543, which was downregulated by 0.21 -fold in endometrial tissue with IUAs. miR-543 was revealed to negatively regulate cadherin 2 , and was detected at significantly increased levels in endometrial tissues with IUAs (12). Cadherin 2 often represents a specific cell lineage transition differentiated from human embryonic stem cells, indicating the likely role of the EMT pathway in cell-to-cell adhesion junctions (28). In the present study, the levels of the epithelial markers, E-cadherin and cytokeratin, were clearly reduced, while levels the mesenchymal marker proteins, $\mathrm{N}$-cadherin and vimentin, were increased in the IUAs group compared with normal tissues. These data suggest that EMT progression is involved in endometrial fibrosis. The biological function of EMT is to produce fibrous cells; if EMT persists in circumstances of activated inflammation, it may eventually lead to organ fibrosis (28). These data may explain the functional role of EMT during endometrial repair following endometrial injury, thus leading to the accumulation of extracellular matrix. By blocking miR-1291 activity following uterine injury, $\alpha$-SMA and collagen 1a levels were reduced and adhesion factors, PDGF-BB and FGF2, were decreased, indicating that the miR-1291 antagomir ameliorated endometrial fibrosis.

ArhGAP29 is a target gene of miR-1291 that exhibits a strong binding affinity for the small GTPase RhoA; inactivation of ArhGAP29 can activate RhoA in an efficient manner (19). Previous studies have demonstrated that RhoA and its effector, ROCK are implicated in several fibrogenic diseases, including those of the lung, kidney, cardiovascular tissues and liver $(25-27,29)$. ROCK1 is a multifunctional kinase that has key roles in a wide range of pathological and physiological processes, including cell apoptosis, proliferation and permeability (30). Selective inhibitors of the RhoA/ROCK1 pathway can thus reduce fibrotic development. ArhGAP29 is a target of miR-1291 and, as a RhoA inactivator, acts upstream of RhoA-ROCK1; thus, we hypothesized that reducing the level of miR-1291 may block the RhoA-ROCK1 pathway and thus reduce the degree of fibrosis. In support of this hypothesis, endometrial tissues in the miR-1291 antagomir group exhibited altered levels of these specific pathway proteins. Immunofluorescence staining, RT-qPCR and western blot analysis demonstrated that endometrial tissues from the miR-1291 antagomir group exhibited increased levels of ArhGAP29, while levels of RhoA and ROCK1 were reduced compared with the IUAs group. It is well established that RhoA is a downstream target of TGF- $\beta$ signaling and that TGF- $\beta$ is a key component in various fibrotic diseases $(31,32)$. However, as we were unable to identify appropriate specific inhibitors for RhoA, ROCK1 or TGF- $\beta$ for use in the current murine model, none of the current experiments tested changes arising from blocking RhoA, ROCK1 or TGF- $\beta$ separately. Therefore, it was not identified how miR-129l regulates or provides feedback for different aspects of the EMT pathway downstream. Future studies are required to elucidate the complex regulation network involved. Crosstalk and interaction between RhoA and TGF- $\beta$ will be assessed by blocking different points in their respective molecular pathways. Furthermore, IUAs are associated with a poor pregnancy prognosis $(2,4)$. The present study did not consider the pregnancy prognosis, as our hypothesis focused specifically upon molecular pathways. Future animal experiments will aim to consider pregnancy prognosis as an important observation.

In summary, a novel role for miR-1291 in regulating endometrial repair was identified. miR-1291 acts on ArhGAP29, which negatively regulates the RhoA-ROCK1 EMT pathway. Thus, by inhibiting miR-1291 it is possible to block the EMT pathway, leading to reduced fibrosis and the amelioration of IUAs. These experiments provide a potential molecular mechanism to explain IUAs pathogenesis. Furthermore, as endometrial fibrosis is the key factor for poor prognosis in patients with IUAs, these studies may provide a new direction for potential diagnostic and therapeutic options. As miR-1291 levels can be detected in the blood plasma, the findings may pave the way to use circulating miRNAs as clinical biomarkers of endometrial fibrosis. However, murine physiology is not identical to that of humans. Therefore, randomized controlled trials in human patients are required in order to validate the clinical importance of the findings of the current study. In addition, it is also necessary to evaluate the administration route and time course of pharmaceutical miRNA agents.

\section{Acknowledgements}

The present study was supported by grants from the National Key Technology Research and Development Program of the Ministry of Science and Technology of China (grant no. 2014BAI05B03), Beijing Municipal Administration of Hospitals Clinical Medicine Development of Special Funding Support (grant no. ZYLX201406) and the Capital Health Research and Development of Special (grant no. 2014-1-2112). 


\section{References}

1. March CM: Asherman's syndrome. Semin Reprod Med 29: 83-94, 2011.

2. Bosteels J, Weyers S, Mol BW and D'Hooghe T: Anti-adhesion barrier gels following operative hysteroscopy for treating female infertility: A systematic review and meta-analysis. Gynecol Surg 11: 113-127, 2014.

3. Deans R and Abbott J: Review of intrauterine adhesions. J Minim Invasive Gynecol 17: 555-569, 2010.

4. Xiao S, Wan Y, Xue M, Zeng X, Xiao F, Xu D, Yang X, Zhang P, Sheng W, Xu J and Zhou S: Etiology, treatment, and reproductive prognosis of women with moderate-to-severe intrauterine adhesions. Int J Gynecol Obstet 125: 121-124, 2014.

5. Expert consensus on the diagnosis and management of intrauterine adhesions in China. Zhonghua Fu Chan Ke Za Zhi 50: 881-887, 2015 (In Chinese).

6. Wei B and Pei G: MicroRNAs: Critical regulators in Th17 cells and players in diseases. Cell Mol Immunol 7: 175-181, 2010.

7. Wu K, Ye C, Lin L, Chu Y, Ji M, Dai W, Zeng X and Lin Y: Inhibiting miR-21 attenuates experimental hepatic fibrosis by suppressing both ERK1 pathway in HSC and hepatocyte EMT. Clin Sci (Lond) 130: 1469-1480, 2016.

8. Bijkerk R, de Bruin RG, van Solingen C, van Gils JM, Duijs JM, van der Veer EP, Rabelink TJ, Humphreys BD and van Zonneveld AJ: Silencing of microRNA-132 reduces renal fibrosis by selectively inhibiting myofibroblast proliferation. Kidney Int 89: 1268-1280, 2016.

9. Wang L, Ma L, Fan H, Yang Z, Li L and Wang H: MicroRNA-9 regulates cardiac fibrosis by targeting PDGFR-beta in rats. J Physiol Biochem 72: 213-223, 2016.

10. Chickooree D, Zhu K, Ram V, Wu HJ, He ZJ and Zhang S: A preliminary microarray assay of the miRNA expression signatures in buccal mucosa of oral submucous fibrosis patients. J Oral Pathol Med 45: 691-697, 2016.

11. Li J, Cen B, Chen S and He Y: MicroRNA-29b inhibits TGF- $\beta 1$-induced fibrosis via regulation of the TGF- $\beta 1 / \mathrm{Smad}$ pathway in primary human endometrial stromal cells. Mol Med Rep 13: 4229-4237, 2016.

12. Liu X, Duan H, Zhang HH, Gan L and Xu Q: Integrated data set of microRNAs and mRNAs involved in severe intrauterine adhesion. Reprod Sci 23: 1340-1347, 2016.

13. Pan YZ, Zhou A, Hu Z and Yu AM: Small nucleolar RNA-derived microRNA hsa-miR-1291 modulates cellular drug disposition through direct targeting of $\mathrm{ABC}$ transporter ABCC1. Drug Metab Dispos 41: 1744-1751,2013.

14. Maurel M, Dejeans N, Taouji S, Chevet E and Grosset CF: MicroRNA-1291-mediated silencing of IRE1 $\alpha$ enhances Glypican-3 expression. RNA 19: 778-788, 2013.

15. Yamasaki T, Seki N, Yoshino H, Itesako $\mathrm{T}$, Yamada $\mathrm{Y}$, Tatarano S, Hidaka H, Yonezawa T, Nakagawa M and Enokida H: Tumor-suppressive microRNA-1291 directly regulates glucose transporter 1 in renal cell carcinoma. Cancer Sci 104: 1411-1419, 2013.

16. Xu K, Sacharidou A, Fu S, Chong DC, Skaug B, Chen ZJ, Davis GE and Cleaver O: Blood vessel tubulogenesis requires Rasip1 regulation of GTPase signaling. Dev Cell 20: 526-539, 2011.
17. Post A, Pannekoek WJ, Ross SH, Verlaan I, Brouwer PM and Bos JL: Rasip1 mediates Rap1 regulation of Rho in endothelial barrier function through ArhGAP29. Proc Natl Acad Sci USA 110: 11427-11432, 2013.

18. Post A, Pannekoek WJ, Ponsioen B, Vliem MJ and Bos JL: Rap1 spatially controls ArhGAP29 to inhibit Rho signaling during endothelial barrier regulation. Mol Cell Biol 35: 2495-2502, 2015.

19. Biggs LC, Naridze RL, DeMali KA, Lusche DF, Kuhl S, Soll DR, Schutte BC and Dunnwald M: Interferon regulatory factor 6 regulates keratinocyte migration. J Cell Sci 127: 2840-2848, 2014.

20. AlawadhiF,Du H,Cakmak Hand TaylorHS: Bone marrow-derived stem cell (BMDSC) transplantation improves fertility in a murine model of Asherman's syndrome. PLoS One 9: e96662, 2014.

21. Livak KJ and Schmittgen TD: Analysis of relative gene expression data using real-time quantitative PCR and the 2 (-Delta Delta C(T)) method. Methods 25: 402-408, 2001.

22. Zhang J, Wang J, Zhou YF, Ren XY, Lin MM, Zhang QQ, Wang YH and Li X: Rich1 negatively regulates the epithelial cell cycle, proliferation and adhesion by CDC42/RAC1-PAK1-Erk1/2 pathway. Cell Signal 27: 1703-1712, 2015.

23. Nakahara S, Tsutsumi K, Zuinen $\mathrm{T}$ and Ohta Y: FilGAP, a Rho-ROCK-regulated GAP for Rac, controls adherens junctions in MDCK cells. J Cell Sci 128: 2047-2056, 2015.

24. McMichael BK, Scherer KF, Franklin NC and Lee BS: The RhoGAP activity of myosin IXB is critical for osteoclast podosome patterning, motility, and resorptive capacity. PLoS One 9: e87402, 2014

25. Bei Y, Hua-Huy T, Nicco C, Duong-Quy S, Le-Dong NN, Tiev KP, Chéreau C, Batteux F and Dinh-Xuan AT: RhoA/Rho-kinase activation promotes lung fibrosis in an animal model of systemic sclerosis. Exp Lung Res 42: 44-55, 2016.

26. Jia Z, Johnson AC, Wang X, Guo Z, Dreisbach AW, Lewin JR, Kyle PB and Garrett MR: Allelic variants in Arhgef11 via the rho-rock pathway are linked to epithelial-mesenchymal transition and contributes to kidney injury in the dahl salt-sensitive rat. PLoS One 10: e132553, 2015.

27. Gao HC, Zhao H, Zhang WQ, Li YQ and Ren LQ: The role of the Rho/Rock signaling pathway in the pathogenesis of acute ischemic myocardial fibrosis in rat models. Exp Ther Med 5: 1123-1128, 2013.

28. D'Amour KA, Agulnick AD, Eliazer S, Kelly OG, Kroon E and Baetge EE: Efficient differentiation of human embryonic stem cells to definitive endoderm. Nat Biotechnol 23: 1534-1541, 2005.

29. Zhang CG, Zhang B, Deng WS, Duan M, Chen W and Wu ZY: Role of estrogen receptor $\beta$ selective agonist in ameliorating portal hypertension in rats with $\mathrm{CCl} 4$-induced liver cirrhosis. World J Gastroenterol 22: 4484-4500, 2016.

30. Olson MF: Applications for ROCK kinase inhibition. Curr Opin Cell Biol 20: 242-248, 2008.

31. Han YY, Shen P and Chang WX: Involvement of epithelial-to-mesenchymal transition and associated transforming growth factor- $\beta /$ Smad signaling in paraquat-induced pulmonary fibrosis. Mol Med Rep 12: 7979-7984, 2015.

32. Korol A, Taiyab A and West-Mays JA: RhoA/ROCK signaling regulates TGF $\beta$-induced epithelial-mesenchymal transition of lens epithelial cells through MRTF-A. Mol Med 22: 713-723 2016. 